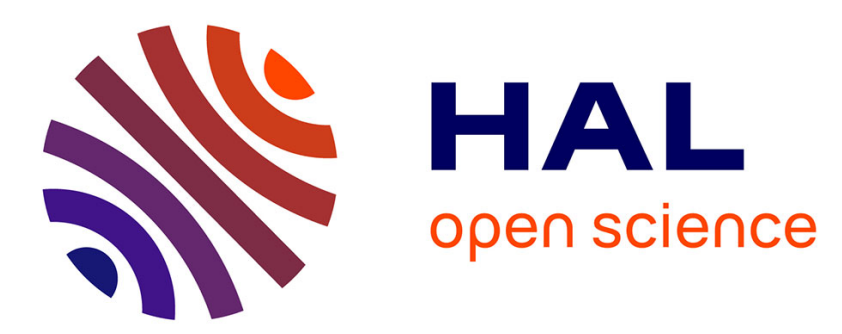

\title{
Towards Chipless RFID Technology based on Micro-Doppler Effect for Long Range Applications
}

\author{
Ashkan Azarfar, Nicolas Barbot, Etienne Perret
}

\section{To cite this version:}

Ashkan Azarfar, Nicolas Barbot, Etienne Perret. Towards Chipless RFID Technology based on MicroDoppler Effect for Long Range Applications. 2021 IEEE/MTT-S International Microwave Symposium - IMS 2021, Jun 2021, Atlanta (virtual), United States. pp.819-822, 10.1109/IMS19712.2021.9575006 . hal-03413160

\section{HAL Id: hal-03413160 https://hal.science/hal-03413160}

Submitted on 3 Nov 2021

HAL is a multi-disciplinary open access archive for the deposit and dissemination of scientific research documents, whether they are published or not. The documents may come from teaching and research institutions in France or abroad, or from public or private research centers.
L'archive ouverte pluridisciplinaire HAL, est destinée au dépôt et à la diffusion de documents scientifiques de niveau recherche, publiés ou non, émanant des établissements d'enseignement et de recherche français ou étrangers, des laboratoires publics ou privés. 


\title{
Towards Chipless RFID Technology based on Micro-Doppler Effect for Long Range Applications
}

\author{
Ashkan Azarfar, Nicolas Barbot, Etienne Perret \\ Univ. Grenoble Alpes, Grenoble INP, LCIS, F-26000 Valence, France \\ ashkan.azarfar@1cis.grenoble-inp.fr
}

\begin{abstract}
The paper describes how chipless RFID tags in motion can be read at higher distances than what is currently done without taking into account displacement. The movement results in the appearance of a tag backscattered field containing frequencies different from those emitted by the reader according to the Doppler effect. The use of these frequencies not generated by the reader considerably increases the reading distance of the tag, especially in a real environment. This paper is focused on a rotational movement of the tag. The proof of concept is carried out on elementary chipless tags which will be read up to $10 \mathrm{~m}$.

Keywords - Chipless RFID, Micro-Doppler, Resonant
\end{abstract} Scatterer.

\section{INTRODUCTION}

Chipless RFID technology has been introduced as an effective solution for cost reduction in RFID market [1]. In this technology, silicon chips, as well as any electronic component, are excluded from the tag structure and so, the identification (ID) data is included in the geometric properties of the tags that are translated into its backscattering behavior [1], [2]. Among different types of frequency coded tags, Radio-frequency Encoding Particles (REP) is the most attractive approach as compact tags can be obtained with higher coding capacity [2]. Indeed, the REPs are composed of microwave resonators, and the ID can be associated to the resonance frequency, as well as to the amplitude, phase, and any polarimetric backscattering properties of each scatterer [2]. According to the best knowledge of the authors, any rigorous study has not been done to investigate the read range limitations of the chipless tags and to discuss about possible solutions for increasing the reading distance. Contrary to classical RFID UHF tags which modify their RCS, chipless tags cannot modulate their backscattered signal since the EM interaction with the chipless tag (and almost all materials in the background environment) can be modeled by linear time-invariant (LTI) systems. Thus, the backscattered signals from the tag and from the background share the same frequencies as the transmitted signal which significantly reduces the read range. Thus, the essential solution to enlarge the read range is to break the linearity or time-invariance of chipless tags.

In radar, the Doppler effect has been studied and used for many applications for decades [3]. The wave backscattered by a moving object will have different frequencies from those emitted by the transmitter. The study of periodic object movements (rotation or vibration in one or several directions) with small displacements with respect to the distance [4], is

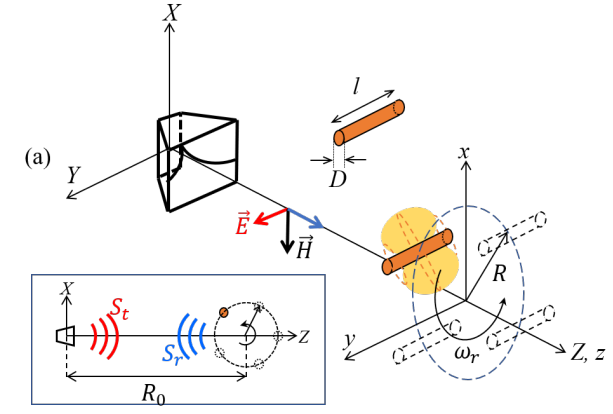

(b)

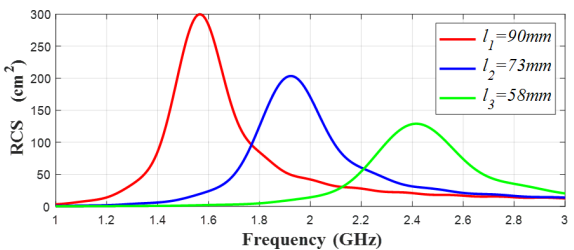

Fig. 1. (a) Principle of the chipless sensor based on micro Doppler effect. (b) Simulated RCS of the dipole scatterer of different length $l$.

known as the micro-Doppler effect [5]. By considering the object exterior surfaces as perfectly conducting surface and large in size with respect to the wavelength, it is possible to establish analytical formulas describing how the backscattered power is distributed among harmonics around the frequency of the incident field [4], [5]. Recently the principle of micro-Doppler effect has been used to realize rotating beacons detectable with automotive FMCW radars [6].

The idea of the paper is to use the Doppler effect on basic chipless tags to allow detection over a reading distance higher than when the tag is stationary. To do this, the micro-Doppler equation is modified to take into account the resonant behaviour of the tags that allows their identification.

\section{Analytical Model}

\section{A. Backscattering from rotating dipole as a chipless tag}

Fig. 1(a) shows a dipole with a length $l$ which is aligned along the $y$-axis and is rotating on a circle with a radius $R$. The rotation angular frequency of the dipole around its rotation axis ( $y$-axis) is $\omega_{r}=2 \pi f_{r}$. The radiated far-field of a $y$-polarized antenna with gain $G_{A}$ is incident on the dipole and the co-polarized scattered field is received by the same antenna. In addition, the induced current on the thin dipole can be perfectly modeled by a line current source with an omni-directional reradiation pattern as shown in 
Fig. 1(a). Thus, the amplitude of the backscattered field does not change during the rotation of the dipole. The phase-varying backscattered fields from the rotating dipole can be accurately modeled using the classical rotation-induced micro-Doppler effect [5]. In the 2D representation of the problem, illustrated in the inset of Fig. 1(a), the dipole can be replaced by a rotating point scatterer in $x z$-plane. For a time-harmonic transmitted wave $s(t)=A \cos \left(2 \pi f_{0} t+\phi_{0}\right)$, with power $P_{t}=A^{2} / 2$, the complex envelope of the periodic received signal can be obtained as [5]:

$$
s_{r}^{c e}(t)=A e^{j \phi_{0}} \rho\left(f_{0}\right) e^{-2 j k\left(R_{0}+R \sin \omega_{r} t\right)}
$$

where, $k=2 \pi f_{0} / c=2 \pi / \lambda, c$ is the velocity of the light, and $\rho\left(f_{0}\right)$ is the frequency dependent reflectivity of the dipole resonator which is utilized here as a REP. Fig. 1(b) shows that the simulated RCS of the dipole depends on its length and so, this frequency dependency has been considered in (1). The periodic signal $s_{r}^{c e}(t)$ can be expressed by a Fourier series in time-domain as:

$$
s_{r}^{c e}(t)=A_{c} \rho\left(f_{0}\right) \sum_{n=-\infty}^{+\infty} J_{n}(\beta) e^{-j n \omega_{r} t}
$$

where $\beta=4 \pi R / \lambda, A_{c}=A e^{j \phi_{0}} e^{-j 2 k R_{0}}$, and $J_{n}(\beta)$ is the Bessel function of the first kind of order $n$. The Fourier transform of (2) gives (3), which clearly describes the newly generated frequency components of $n f_{r}$ due to the micro-Doppler effect, which is the vital point to increase the read range of the proposed rotating chipless tag.

$$
S_{r}^{c e}(f)=A_{c} \rho\left(f_{0}\right) \sum_{n=-\infty}^{+\infty} J_{n}(\beta) \delta\left(f-n f_{r}\right)
$$

\section{B. Differential RCS for rotating chipless tag}

Differential RCS has been introduced in [7] to determine the power of the modulated tag signal. Originally defined for UHF tags, this definition has however been extended to any modulating tag [8] and can be applied to the tags presented in this paper. The delta RCS can be computed directly from (3) while remarking that the power associated to the real signal is half of the one associated to the complex envelope:

$$
\begin{gathered}
\sigma_{d}\left(f_{0}\right)=\frac{(4 \pi)^{3} R_{0}^{4}}{2 P_{t} G_{A}^{2} \lambda^{2}}\left(\int_{-\infty}^{-\epsilon}\left|S_{r}^{c e}(f)\right|^{2} \mathrm{~d} f+\int_{+\epsilon}^{+\infty}\left|S_{r}^{c e}(f)\right|^{2} \mathrm{~d} f\right) \\
\sigma_{d}\left(f_{0}\right)=\sigma\left(f_{0}\right)\left[1-J_{0}^{2}(\beta)\right]
\end{gathered}
$$

where $2 \epsilon$ is the minimal bandwidth which allows to remove the power at $f=0$, and $\sigma\left(f_{0}\right)=\left(\left|\rho\left(f_{0}\right)\right|^{2}(4 \pi)^{3} R_{0}^{4}\right) /\left(G_{A}^{2} \lambda^{2}\right)$ is the RCS of the short-circuited dipole at the frequency $f_{0}$. Note that (5) has been obtained using the well-known equality $\sum_{n=-\infty}^{+\infty}=J_{n}^{2}(\beta)=1$. This relation allows to link the classical RCS to the delta RCS.

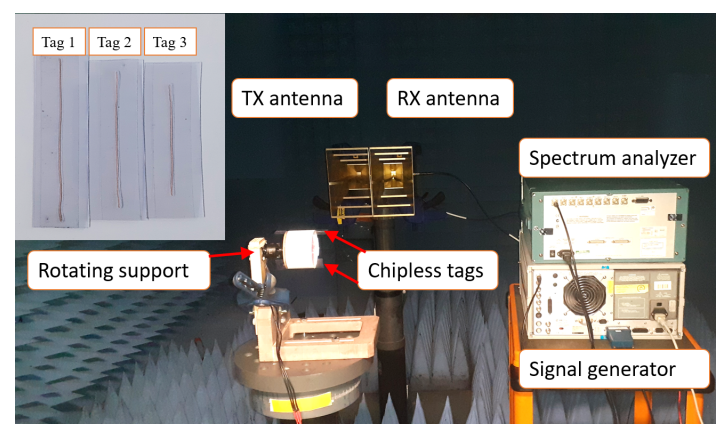

Fig. 2. Measurement bench used to characterize the micro-Doppler effect generated by the rotation of the tags. Dipole scatterers used in the study with length of 90,73 and $58 \mathrm{~mm}$ and $D=100 \mu \mathrm{m}$ are shown in inset.

\section{RESUlt AND Discussion}

\section{A. Measurement Bench}

Measurement bench is presented in Fig. 2 and is composed of a VNA (HP 8720D) and a spectrum analyzer (Tektronix RSA3408A). Both instruments are respectively connected to the transmitting and receiving antennas (A.H. Systems, INC. SAS-571). Antennas have been placed in close proximity to tend to a monostatic configuration. Note that the VNA operates in CW mode and only acts as a RF source. Frequency can be set in-between 1 and $2.6 \mathrm{GHz}$ and the output power has been set to $0 \mathrm{dBm}$. The spectrum analyzer acquires the backscattered signal over a span of $1 \mathrm{kHz}$ around the $\mathrm{CW}$ generated by the VNA. Also, both instruments are synchronized using the same $10 \mathrm{MHz}$ reference signal. Tags are placed on a rotating support of radius $3.7 \mathrm{~cm}$ and rotate at a speed of 70 turns $/ \mathrm{sec}$. The rotating support is placed at a distance of $1 \mathrm{~m}$ from the antennas to fulfill far-field condition.

Chipless tags considered in this paper are also presented in the inset of Fig. 2 and correspond to short-circuited dipole scatterers. Three tags have been designed using a $100 \mu \mathrm{m}$ copper wire. To increase mechanical robustness, tags have been encapsulated in-between 2 layers of PET.

\section{B. Backscattered Power Spectral Density}

The backscattered PSD generated by the rotating tag is presented in Fig. 3. Since the environment is a LTI system, the received PSD (when the tag is not rotating) is only located at $f_{0}$ (or $f=0$ after the demodulation done by the spectrum analyzer). When the tag is rotating, peaks located at $n f_{r}$ with an amplitude weighted by the Bessel function as predicted by (3) is observed, thus model and measurement are in a good agreement. Also, each tag can be differentiated using the amplitude of the backscattered sidelobes. For example, in Fig. 3 with $f_{0}=1.75 \mathrm{GHz}$, Tag 2 has an amplitude which is higher than Tag 1 and 3. The same trend as seen in the RCS [see Fig. 1(b)] is observed at this frequency.

\section{Identification}

In Fig. 3, the PSD is clearly different form one tag to another. However, this way to identify each tag implies that all the tags have to be placed at the same distance from 


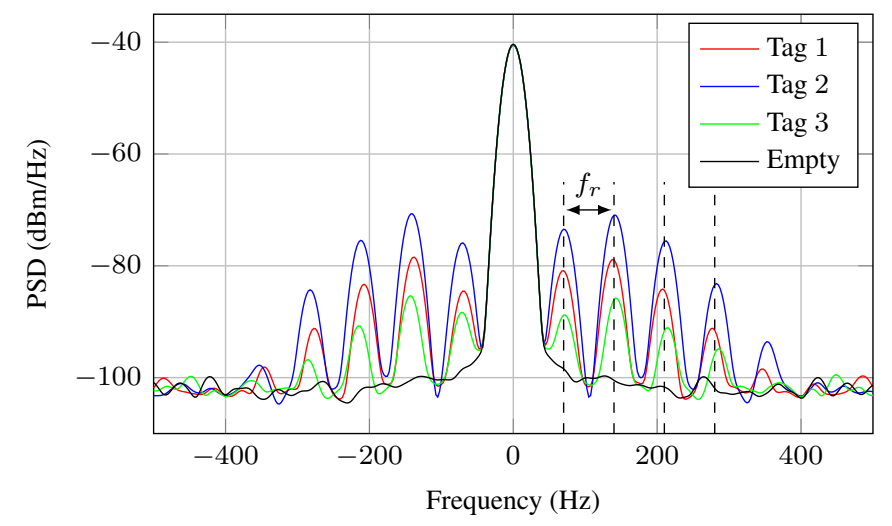

Fig. 3. Measurement of the backscattered power spectral density for the 3 tags rotating at $f_{r}=70 \mathrm{~Hz}$ for a carrier frequency $f_{0}$ of $1.75 \mathrm{GHz}$. Empty corresponds to the PSD of the chamber when no object is rotating.

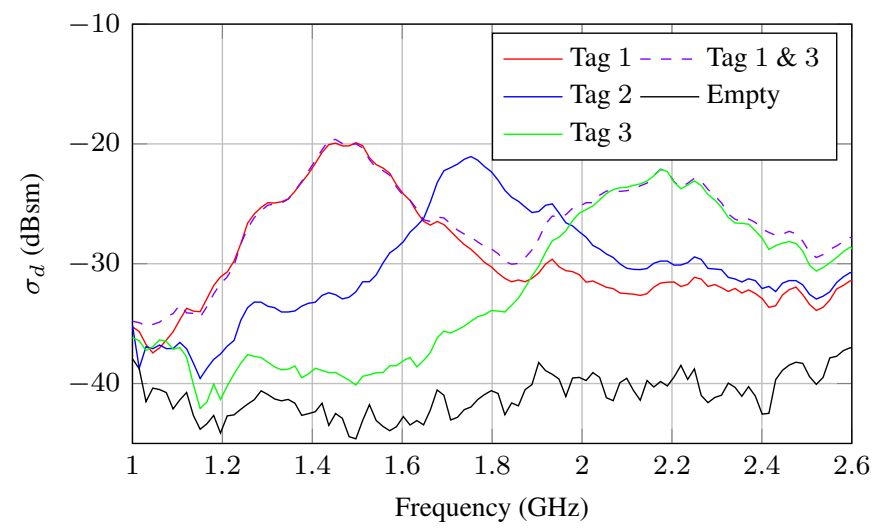

Fig. 4. Delta RCS as a function of the carrier frequency for the 3 rotating tags computed using measured backscattered PSD. Empty corresponds to the delta RCS of the chamber when no object is rotating.

the antennas. If distance is changed, the amplitude of the backscattered signal is also changed which makes the tag identification impossible. The variation of the delta RCS $\sigma_{d}$ as a function of the frequency, given by (5), can be used as a metric which is independent of the measurement distance. Fig. 4 presents $\sigma_{d}$ as a function of the carrier frequency; it presents a maximum at the resonant frequency of the dipole as it was predicted with (5) and Fig. 1(b). Also, the 3 resonant frequencies can clearly be separated which shows that the 3 tags can be identified only using the position of the peaks. Note that tags delta RCS have been obtained without using any empty measurement.

Finally, the dashed line presents the delta RCS when Tag 1 and 3 are placed (at the same time) on the rotating support. In this case, we can see that both resonators can be simultaneously identified. Thus, the design of a rotating chipless tag composed of several resonators can be realized [1], [2]. Coding capacity for this multi-resonator tag, assuming that the ID is coded with presence or absence of a given resonator can be estimated by dividing the total bandwidth $(1.6 \mathrm{GHz})$ by the bandwidth of a single resonator $(400 \mathrm{MHz})$. Thus, the proposed multi-resonator tag has a capacity of 4 bits in the

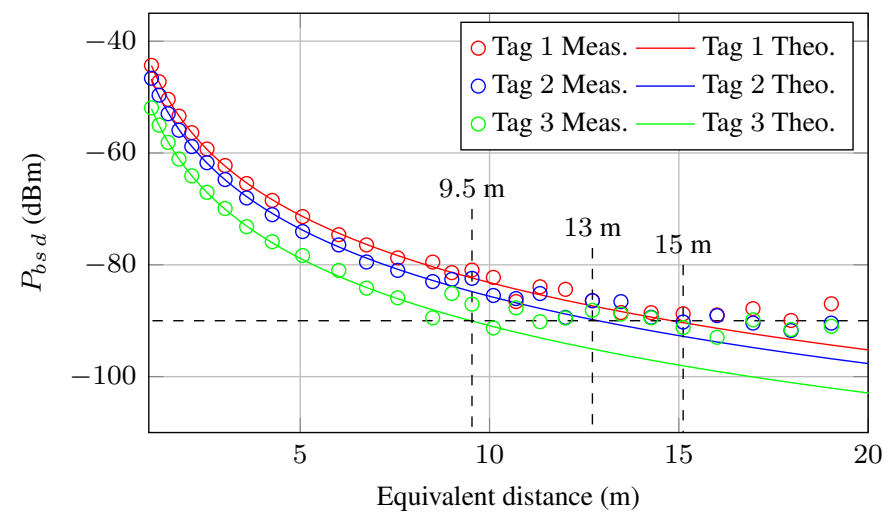

Fig. 5. Read range of the 3 different tags as a function of the equivalent distance considering a carrier frequency of $1.45,1.75$ and $2.18 \mathrm{GHz}$ for Tag 1,2 and 3 respectively. Noise floor of the system is observed at $-90 \mathrm{dBm}$.

bandwidth 1 to $2.6 \mathrm{GHz}$.

\section{Read Range}

As presented in [1], classical chipless tags are usually characterized by a read range lower than $30 \mathrm{~cm}$ since both tag and environment are LTI systems. However, read range of the presented rotating tag can be determined using a modified radar equation obtained by replacing $\sigma$ by $\sigma_{d}$ [see (5)].

Modulated power $P_{b s d}$ (i.e., power associated to the side lobes) has been measured as a function of the transmitted power $P_{t}$ in the interval $[-50 ;+5] \mathrm{dBm}$ at a fixed distance of $1 \mathrm{~m}$ in anechoic chamber. For each $P_{t}$ value, the equivalent distance has been determined, assuming that a fixed value of $+5 \mathrm{dBm}$ has been used in by the VNA. Results are presented in Fig. 5 where we see that $P_{b s d}$ is a decreasing function of the equivalent distance. Theoretical $P_{b s d}$ considering the delta RCS estimated from Fig. 4 has also been added to the plot and presents a good agreement with the measured data. For high equivalent distances, measured power remains constant around $-90 \mathrm{dBm}$ due to the noise floor of the instrument. Read range of the rotating tags can thus been obtained by finding the distance at which the modulated power is higher than the noise floor. Results depends on the tag delta RCS and are equal to 15,13 and $9.5 \mathrm{~m}$ for Tag 1,2, and 3 respectively. These read ranges outperform the ones associated to classical chipless tag detection by a factor of 30 .

\section{Conclusion}

Identification of chipless tags exploiting the Doppler effect is introduced for the first time. A model taking into account the frequency behavior of the tag has been developed. The delta RCS of the tag has been obtained analytically and measured. The results are in accordance with the theory and show that reading distances higher that $10 \mathrm{~m}$ can be achieved. A rotational movement of the tag was studied for ease of implementation. However, the approach is not limited to this type of movement and this principle can be used on tags moving at a lower speed, in the case of translations for instance when the tagged objects are positioned on a conveyor belt. 


\section{ACKNOWLEDGMENT}

This work was supported by the European Research Council (ERC) through the European Union's Horizon 2020 Research and Innovation Program (ScattererID) under Grant $\mathrm{N}^{\circ} 772539$.

\section{REFERENCES}

[1] E. Perret, Radio frequency identification and sensors: from RFID to chipless RFID. John Wiley \& Sons, 2014.

[2] A. Vena, E. Perret, and S. Tedjini, Chipless RFID based on RF encoding particle: realization, coding and reading system. Elsevier, 2016.

[3] V. C. Chen, The micro-Doppler effect in radar. Artech House, 2019.

[4] R. Kleinman and R. Mack, "Scattering by linearly vibrating objects," IEEE Trans. Antennas Propag., vol. 27, no. 3, pp. 344-352, May 1979.

[5] V. C. Chen, F. Li, S. . Ho, and H. Wechsler, "Micro-Doppler effect in radar: phenomenon, model, and simulation study," IEEE Trans. Aerosp. Electron. Syst., vol. 42, no. 1, pp. 2-21, Jan. 2006.

[6] A. Lazaro, M. Lazaro, R. Villarino, and P. De Paco, "New radar micro-doppler tag for road safety based on the signature of rotating backscatters," IEEE Sensors J., vol. 21, no. 6, pp. 8604-8612, Mar. 2021.

[7] P. V. Nikitin, K. V. S. Rao, and R. D. Martinez, "Differential RCS of RFID tag," Electron. Lett., vol. 43, no. 8, pp. 431-432, Apr. 2007.

[8] N. Barbot, O. Rance, and E. Perret, "Differential RCS of modulated tag," IEEE Trans. Antennas Propag., early access. 